\title{
Urinary N-Acetyl- $\beta$-d-Glucosaminidase (NAG) Levels and Risk of Cardiovascular Events in Diabetic Patients
}

\author{
Weiwei Lou' \\ Qun Cheng (D) \\ Yanqiu Liang' \\ Ding $\mathrm{Xia}^{2}$ \\ 'Department of Rehabilitation, Tongji \\ Hospital, Huazhong University of Science \\ and Technology, Wuhan, Hubei, 430030, \\ People's Republic of China; ${ }^{2}$ Urology \\ Surgery, Tongji Hospital, Tongji Medical \\ College of Huazhong University of \\ Science and Technology, Wuhan, Hubei, \\ 430030, People's Republic of China
}

Background: Cardiovascular diseases (CVDs) have a high incidence rate in population with diabetic patients. Studies on the association between urinary N-acetyl- $\beta$-d-glucosaminidase (NAG) levels, the biomarker of renal tubular damage, with cardiovascular (CV) events diabetic patients was still few.

Methods: The relationship between urinary NAG levels and CV events was analyzed in a prospective cohort including 357 patients with type 2 diabetes mellitus at a follow-up of 5 years.

Results: Twenty-six (7.3\%) patients have CV events. Kaplan-Meier analysis suggested that diabetic patients with urine NAG levels $\geq 37.5 \mathrm{IU} / \mathrm{L}$ had a higher rate of CV events than those with urine NAG levels $<37.5 \mathrm{IU} / \mathrm{L}$ (Log rank test, $\mathrm{P}=0.021)$. Cox analysis revealed that elevated urine NAG levels significantly contributed to increased risk of CV events (HR = $1.43,95 \%$ CI 1.23-1.93, $P<0.001)$ after adjusting for clinical confounding factors. Interestingly, we also found that "abnormal renal function" has an effect modification on the association between urine NAG levels and CV events. ROC-AUC analysis suggested that the urine NAG $(\mathrm{AUC}=0.81, \mathrm{P}<0.001)$ had a better predictive value than eGFR (AUC = $0.74, \mathrm{P}=0.012)$.

Conclusion: Elevated urine NAG levels are associated with higher risk of CV events in patients with type 2 diabetes. These results might further suggested that urinary NAG is a value urinary biomarker for early detecting $\mathrm{CV}$ events among diabetic patients.

Keywords: N-acetyl- $\beta$-d-glucosaminidase, type 2 diabetes mellitus, cardiovascular event, diabetic nephropathy

\section{Introduction}

Cardiovascular (CV) events, such as coronary artery disease, heart failure, stroke, cardiac arrhythmia and peripheral vascular diseases, are the main causes of morbidity and mortality among diabetic patients. ${ }^{1-3}$ Compared with patients without diabetes, the incidence rate of $\mathrm{CV}$ events increased by 2-4 times in diabetic patients. ${ }^{4}$ Hence, developing methods with more accuracy to detecting the risk of $\mathrm{CV}$ events is necessary to reduce $\mathrm{CV}$ mortality and improve prognosis for diabetic patients.

Some surrogate measures, like pulse wave velocity (PWV) and albuminuria, predicting $\mathrm{CV}$ events have been investigated. Existing studies showed that PWV has been considered as the gold standard for detecting arterial stiffness and is significantly associated with increased risk of atherosclerosis-related pathological
Department of Urology, Tongji Hospital, 1095 Jiefang Ave, Wuhan, Hubei, 430030 People's Republic of China

Tel +8618177494319

Email dingxia I23I23@I26.com 
deterioration such as increased intima-media thickness of carotid artery and the presence of carotid plaques, ${ }^{5,6}$ and $\mathrm{CV}$ outcomes such as coronary artery disease and stroke. ${ }^{7,8}$ As an indicator of early renal injury, albuminuria has been regarded as a biomarker for determining diabetic kidney disease, suggesting positive independent associations with risk of cardiovascular disease (CVD) and mortality. ${ }^{9}$ Interestingly, some recent studies reported significant associations between albuminuria and macrovascular complications including coronary artery calcification and PWV in patients with essential hypertension or diabetes mellitus. ${ }^{9,10}$ However, the association between urinary $\mathrm{N}$-acetyl- $\beta$-d-glucosaminidase (NAG), a marker of renal tubulopathy, and $\mathrm{CV}$ events and in patients with type 2 diabetes mellitus is still controversial. The controversy maybe originated from different characteristics of study populations including age, gender, ethnic diversity and duration of diabetes, as well as different methods used to measure $\mathrm{CV}$ events. ${ }^{10}$

In recent years, several biomarkers of renal tubular damage have attracted more and more attention due to their predictive value on predicting progression of earlystage diabetic kidney disease. ${ }^{11-13}$ Injury of proximal renal tubular cells mainly contributes to increased level of urine. Furthermore, increases in urinary NAG has been found in mildly increased albuminuric patients with type 2 diabetes mellitus. ${ }^{11}$ Some studies have also suggested that urinary NAG levels were correlated with macrovascular disease, ${ }^{14}$ neuropathy ${ }^{15}$ and retinopathy. ${ }^{16}$ However, few or controversial study has focused on the association between urinary NAG levels and CV outcomes in patients with type 2 diabetes mellitus. We would like to investigate the relationship between urinary NAG and CV events in patients with type 2 diabetes mellitus.

\section{Methods}

We continuously included 377 hospitalized patients with type 2 diabetes mellitus who are from clinical research center from April 2012 to December 2014 by using a prospective design. These patients have also been detected for blood glucose parameters, urinary NAG, and have undergone cardiovascular examination (ECG, echocardiography and others) after admission. All included patients with type 2 diabetes mellitus did not have any CVD history and other serious chronic diseases (cancers, severe liver disease, active infectious disease and connective tissue disease) before admission. The definition for type 2 diabetes mellitus was based on the basis: ${ }^{17} 1$ ) when fasting plasma glucose $>7.0 \mathrm{mmol} / \mathrm{L}$, diabetes can be diagnosed if the typical symptoms of diabetes; 2) when 2-hour postprandial blood glucose $>11.1 \mathrm{mmol} / \mathrm{L}$, if accompanied by typical symptoms of diabetes and 3) clinically, in the case of reliable test methods, glycosylated hemoglobin (HbA1C) can also be used for the diagnosis of type 2 diabetes mellitus when $\mathrm{HbA} 1 \mathrm{C}>6.5 \%$. Furthermore, in order to achieve the purpose of the study, 1) the taking hypoglycemic therapy liking using insulin and oral hypoglycemic agents was defined type 2 diabetes mellitus. Additionally, patients were excluded from this cohort study due to an CVD history including coronary artery disease $(\mathrm{N}=13)$ and stroke $(\mathrm{N}=7)$ and other disease including cancers $(\mathrm{N}=5)$, severe liver disease $(\mathrm{N}=3)$, active infectious disease $(\mathrm{N}=1)$ and connective tissue disease $(\mathrm{N}=1)$ before admission. Clinical variables including age, gender, body mass index (BMI), smoking and drinking habits, systolic and diastolic blood pressure, diabetes duration, medical history and others were recorded. BMI was defined as weight divided by height squared $\left(\mathrm{kg} / \mathrm{m}^{2}\right)$. The institutional review board of Tongji Hospital approved this study and all included patients gave the written informed consent, consistent with Declaration of Helsinki guidelines.

Finally, all included patients type 2 diabetes mellitus $(\mathrm{N}=357)$ were followed up by telephone until the occurrence of $\mathrm{CV}$ events. If the status of these patients was not clear completely during the follow-up, referring cardiologists were invited to identify the patient's condition. During a mean follow-up of 5 years, $\mathrm{CV}$ events requiring hospitalization were defined by acute myocardial infarction $(\mathrm{N}=12)$, ischemic or hemorrhagic stroke $(\mathrm{N}=7)$, acute HF $(\mathrm{N}=5)$ and death $(\mathrm{N}=2)$ caused by these diseases. Twelve diabetic patients lost contact during a mean follow-up of 5 years. CV events occurred in 26 diabetic patients.

\section{Measurement of Blood and Urine Markers}

Urine samples of fasting morning were obtained from each included patient with type 2 diabetes mellitus and were measured for urinary NAG levels using a JCA-BM 6010/c automated chemistry analyzer (JEOL Ltd., Tokyo, Japan). Fasting blood samples from each included patient after admission blood were also tested for creatinine, uric acid, glucose, $\mathrm{HbA1C}$, hemoglobin and lipid profiles. Measurement of HbAlc was performed by an Integra 
800 CTS (Roche, Hercules, CA, USA). Serum lipid levels of profiles were measured by an enzymatic method (Asahi Kasei Pharma Co, Tokyo, Japan). Serum creatinine, uric acid, hemoglobin and glucose were tested by the Hitachi 7600 analyzer (Hitachi Ltd.). The estimated glomerular filtration rate (eGFR) was derived from the Chronic Kidney Disease Epidemiology Collaboration (CKD-EPI). ${ }^{18}$ eGFR $<60 \mathrm{~mL} / \mathrm{min}$ per $1.73 \mathrm{~m}^{2}$ was defined as renal function injury.

\section{Statistical Analyses}

All of the data were analyzed by using SPSS 25.0. $P \leq$ 0.05 was considered to be statistically significant. A $\mathrm{K}-\mathrm{S}$ test was performed for normality of all continuous variables. Baseline characteristics upon entry into the cohort were compared between tertiles of eGFR $\left(<60 \mathrm{~mL} / \mathrm{min}\right.$ per $1.73 \mathrm{~m}^{2}$ and $\geq 60 \mathrm{~mL} / \mathrm{min}$ per $1.73 \mathrm{~m}^{2}$ ). In multivariate analysis, no adjustment was made for confounding factors in Crude model.
Adjustments of confounding factors including age, gender, smoking history, drinking history and BMI were made in Model 1. In Model 2, adjustments of age, gender, smoking history, current drinking history, BMI, systolic blood pressure, diastolic blood pressure and moderate physical activity were made.

Cox regression analysis was performed to identify the independent predictive value of urine NAG levels for $\mathrm{CV}$ events in patients with type 2 diabetes mellitus. To further evaluate the independent association, we further excluded the effect of "duration of diabetes" by sensitivity analysis. We also analyzed the association between urine NAG levels at baseline and $\mathrm{CV}$ events during the follow-up by stratified analysis by adding "eGFR $\geq 60 \mathrm{~mL} / \mathrm{min}$ per $1.73 \mathrm{~m}^{2}$ " as the covariate. Finally, the performance of urine NAG for predicting $\mathrm{CV}$ events was analyzed by receiver operating characteristic (ROC) curves and area under the curve (AUC).

Table I Clinical Characteristics in Patients with Type 2 Diabetes Mellitus $(\mathrm{N}=357)$

\begin{tabular}{|c|c|c|c|}
\hline Characteristic & $\begin{array}{c}\text { eGFR } \geq 60 \mathrm{~mL} / \text { Min per } 1.73 \mathrm{~m}^{2} \\
(\mathrm{~N}=178)\end{array}$ & $\begin{array}{c}\text { eGFR }<60 \mathrm{~mL} / \text { Min per } 1.73 \mathrm{~m}^{2} \\
(\mathrm{~N}=179)\end{array}$ & $P$ value \\
\hline Age $(Y)$ & $68.3 \pm 9.6$ & $70.5 \pm 10.6$ & 0.009 \\
\hline Male, n (\%) & $105(58.9)$ & $102(57.0)$ & 0.158 \\
\hline CV events, n (\%) & $8(4.5)$ & $18(10.1)$ & $<0.001$ \\
\hline Duration of diabetes (years) & $6.9(4.1-12.4)$ & $11.9(7.3-13.3)$ & $<0.001$ \\
\hline Smoking history, n (\%) & $64(36.0)$ & $7 \mid(39.7)$ & 0.045 \\
\hline Drinker history, n (\%) & $102(57.3)$ & III (62.0) & 0.052 \\
\hline BMI $\left(\mathrm{kg} / \mathrm{m}^{2}\right)$ & $27.3 \pm 6.3$ & $29.5 \pm 7.1$ & $<0.001$ \\
\hline Systolic blood pressure $(\mathrm{mmHg})$ & $|46.4 \pm| \mid .8$ & $155.5 \pm 16.4$ & $<0.001$ \\
\hline Diastolic blood pressure $(\mathrm{mmHg})$ & $83.1 \pm 9.3$ & $87.5 \pm 9.8$ & $<0.001$ \\
\hline Moderate physical activity, n (\%) & $66(37.1)$ & $72(40.2)$ & 0.096 \\
\hline Urinary NAG (IU/L) & $32.6 \pm 4.2$ & $4 I \pm 4.8$ & $<0.001$ \\
\hline Urinary NAG (U/g creatinine) & $4.3(2.1-7.2)$ & $12.6(8.4-17.2)$ & $<0.001$ \\
\hline Estimated GFR (mL/min per $1.73 \mathrm{~m} 2)$ & $70.6 \pm 5.7$ & $48.4 \pm 6.9$ & $<0.001$ \\
\hline Serum albumin $(g / L)$ & $43.4 \pm 3.1$ & $38.3 \pm 3.6$ & $<0.001$ \\
\hline Serum hemoglobin $(\mathrm{g} / \mathrm{L})$ & $115 \pm 19$ & $108 \pm 19$ & 0.079 \\
\hline Serum C-reactive protein (mg/L) & $1.9(0.6-7.6)$ & $7.3(1.1-12.5)$ & $<0.001$ \\
\hline Serum total cholesterol (mmol/L) & $5.1 \pm 0.2$ & $5.4 . \pm 0.3$ & 0.134 \\
\hline Serum triglycerides (mmol/L) & $3.4 \pm 0.2$ & $3.5 \pm 0.4$ & 0.115 \\
\hline Serum HDL cholesterol (mmol/L) & $1.4 \pm 0.1$ & $1.6 \pm 0.2$ & $<0.097$ \\
\hline Serum LDL cholesterol (mmol/L) & $1.6 \pm 0.4$ & $1.7 \pm 0.5$ & 0.231 \\
\hline Serum creatinine $(\mathrm{mmol} / \mathrm{L})$ & $68 \pm 5$ & $135 \pm 1 \mid$ & $<0.001$ \\
\hline Serum uric acid (mmol/L) & $288 \pm 10$ & $305 \pm 13$ & 0.042 \\
\hline Hemoglobin AIc (\%) & $6.8 \pm 0.3$ & $6.9 \pm 0.2$ & 0.344 \\
\hline Fasting plasma glucose (mmol/L) & $8.5 \pm 0.2$ & $8.6 \pm 0.4$ & 0.403 \\
\hline
\end{tabular}

Notes: Data are presented as mean \pm SD for normally distributed data, as median (interquartile range) for nonnormally distributed data, and as $\mathrm{n}$ (\%) for categoric variables. Abbreviations: NAG, N-acetyl- $\beta$-d-glucosaminidase; CV, cardiovascular; BMI, body mass index; eGFR, estimated glomerular filtration rate; HDL, high density lipoprotein; LDL, low density lipoprotein. 
Table 2 Multivariate Cox Regression Analysis of Predicting CV Events in Patients with Type 2 Diabetes Mellitus (N = 357)

\begin{tabular}{|l|c|c|c|}
\hline & Crude & Model I & Model 2 \\
\hline Estimated GFR (mL/min per $\left.1.73 \mathrm{~m}^{2}\right)$ & $1.42(1.22-1.76)$ & $1.36(1.18-1.76)$ & $1.32(1.14-1.68)$ \\
P value & 0.017 & 0.019 & 0.021 \\
Urinary NAG (IU/L) & $1.59(1.31-1.98)$ & $1.47(1.26-1.89)$ & $1.43(1.23-1.93)$ \\
P value & 0.009 & 0.010 & 0.015 \\
Urinary NAG (U/g creatinine) & $1.64(1.39-2.62)$ & $1.57(1.34-2.41)$ & $1.51(1.31-2.27)$ \\
P value & 0.002 & 0.007 & 0.010 \\
\hline
\end{tabular}

Notes: Model I: Adjusted for no. Model 2: Adjusted for age, gender, smoking history, drinking history and BMI. Model 3: Adjusted for age, gender, smoking history, drinking history, BMI, systolic blood pressure, diastolic blood pressure and moderate physical activity.

Abbreviations: NAG, N-acetyl- $\beta$-d-glucosaminidase; CV, cardiovascular; BMI, body mass index; eGFR, estimated glomerular filtration rate.

\section{Results}

\section{Clinical Characteristics of Patients with Type 2 Diabetes Mellitus}

The clinical features of patients with type 2 diabetes mellitus are described in Table 1. These included patients were divided into two groups according to renal function injury $\left(\mathrm{eGFR}<60 \mathrm{~mL} / \mathrm{min}\right.$ per $\left.1.73 \mathrm{~m}^{2}\right)$. Compared with diabetic patients with normal renal function (eGFR $\geq 60 \mathrm{~mL} / \mathrm{min}$ per $1.73 \mathrm{~m}$ ), patients with renal function injury had a higher rate of CV events and higher urine NAG levels, which initially suggested that elevated urine NAG levels were potentially associated with an increased risk of CV events. Furthermore, these patients with renal function injury showed a higher age, BMI, duration of diabetes, systolic blood pressure and diastolic blood pressure and tended to have higher rate of smoking history. Information about laboratory indexes and medications was also described in detail (Table 1).

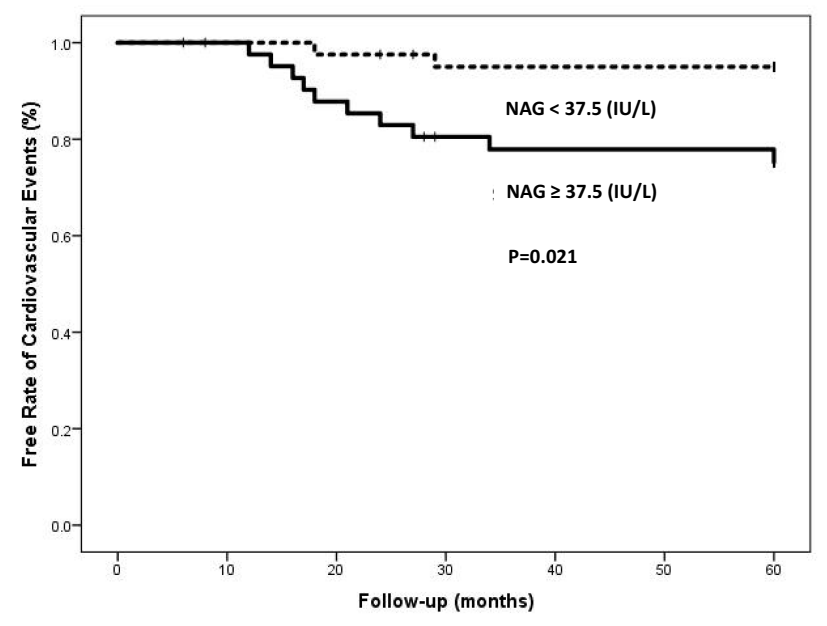

Figure I Kaplan-Meier analysis for cardiovascular events free stratified by urine NAG.

\section{Elevated Urine NAG Levels Were Independently Related to CV Events in Patients with Type 2 Diabetes Mellitus}

To investigate the association of urine NAG levels with $\mathrm{CV}$ events in patients with type 2 diabetes, Cox regression analysis was performed. Cox analysis revealed that elevated urine NAG levels significantly contributed to increased risk of $\mathrm{CV}$ events $(\mathrm{HR}=1.43,95 \%$ CI $1.23-$ $1.93, P<0.001$, Table 2) after adjustments for age, gender, smoking history, drinking history, BMI, systolic blood pressure, diastolic blood pressure and moderate physical activity were made in Model 3. Kaplan-Meier analysis suggested that diabetic patients with urine NAG levels $\geq$ 37.5 IU/L had a higher rate of CV events than those with urine NAG levels $<37.5 \mathrm{IU} / \mathrm{L}$ ( $\log$ rank test, $\mathrm{P}=0.021$, Figure 1).

To further clarify the association between urine NAG levels and $\mathrm{CV}$ events in patients with type 2 diabetes, an additional sensitivity analysis was performed by adding "duration of diabetes" as a covariate. Our results still showed that elevated urine NAG levels were still significantly associated with a higher risk of $\mathrm{CV}$ events ( $\mathrm{HR}=$ $1.27,95 \%$ CI $1.18-1.72, P=0.023$, Table 2 ) after adjusting age, gender, smoking history, drinking history, BMI, systolic blood pressure, diastolic blood pressure, moderate physical activity and duration of diabetes in Model 3 (Table 3).

\section{Elevated Urine NAG Levels Were Independently Related to CV Events by Stratified Analysis in Patients with Type 2 Diabetes Mellitus}

To confirm whether "eGFR $\geq 60 \mathrm{~mL} / \mathrm{min}$ per $1.73 \mathrm{~m}^{2 \text { " }}$ have an effect modification on the independent association 
Table 3 Sensitivity Analysis of Predicting CV Events in Patients with Type 2 Diabetes Mellitus By Adding "Duration of Diabetes" as the covariate $(\mathrm{N}=357)$

\begin{tabular}{|c|c|c|c|}
\hline & Crude & Model I & Model 2 \\
\hline Estimated GFR $\left(\mathrm{mL} / \mathrm{min}\right.$ per $\left.1.73 \mathrm{~m}^{2}\right)$ & $1.32(1.21-1.73)$ & $1.25(1.18-1.69)$ & $1.23(1.13-1.64)$ \\
\hline$P$ value & 0.035 & 0.40 & 0.41 \\
\hline Urinary NAG (IU/L) & $1.38(1.25-1.84)$ & $1.33(1.21-1.76)$ & $1.27(1.18-1.72)$ \\
\hline$P$ value & 0.011 & 0.018 & 0.023 \\
\hline Urinary NAG (U/g creatinine) & $1.43(1.27-2.35)$ & $1.38(1.25-2.14)$ & $1.35(1.23-2.09)$ \\
\hline$P$ value & 0.007 & 0.009 & 0.012 \\
\hline
\end{tabular}

Notes: Model I: Adjusted for duration of diabetes. Model 2: Adjusted for age, gender, smoking history, drinking history, BMI, duration of diabetes and duration of diabetes. Model 3: Adjusted for age, gender, smoking history, drinking history, BMI, systolic blood pressure, diastolic blood pressure, moderate physical activity and duration of diabetes.

Abbreviations: NAG, $\mathrm{N}$-acetyl- $\beta$-d-glucosaminidase; CV, cardiovascular; BMI, body mass index; eGFR, estimated glomerular filtration rate.

between urine NAG levels and CV events. These included patients were divided into two groups according to renal function injury (eGFR $\geq 60 \mathrm{~mL} / \mathrm{min}$ per $1.73 \mathrm{~m}^{2}$ ). In diabetic patients with eGFR $\geq 60 \mathrm{~mL} / \mathrm{min}$ per $1.73 \mathrm{~m}^{2}$, Our found that urine NAG levels did not associate with $\mathrm{CV}$ events $(\mathrm{HR}=1.04,95 \% \mathrm{CI} 0.89-1.47, P=0.157)$ in diabetic patients (Table 4). However, in diabetic patients with eGFR $<60 \mathrm{~mL} / \mathrm{min}$ per $1.73 \mathrm{~m}^{2}$, we still found that elevated urine NAG levels significantly contributed to increased risk of $\mathrm{CV}$ events $(\mathrm{HR}=1.50,95 \%$ CI $1.27-$ 2.96, $P=0.016)$.

\section{ROC Analysis of Predicting CV Events in Patients with Type 2 Diabetes Mellitus}

To determine performance of urine NAG levels for predicting CV events, ROC-AUC analysis was used (Table 5).
The urine NAG $(\mathrm{AUC}=0.81, \mathrm{P}<0.001)$ had a better predictive value than eGFR (AUC $=0.74, \mathrm{P}=0.012)$. The result suggested that urine NAG might be a valuable biomarker for predicting $\mathrm{CV}$ event in patients with type 2 diabetes mellitus.

\section{Discussion}

The prevalence of diabetes mellitus has been increasing remarkably around the world and cardiovascular, neurological and renal complications are the main causes of death in diabetic patients. ${ }^{1-3}$ For example, diabetes can lead to kidney damage and even kidney failure even if blood glucose levels are not controlled. ${ }^{19}$ In recent years, several biomarkers of renal damage have attracted more and more attention due to their predictive value on predicting progression of early-stage diabetic kidney disease. Markers of

Table 4 Stratified Analysis of Predicting CV Events in Patients with Type 2 Diabetes Mellitus By Adding "eGFR $\geq 60 \mathrm{~mL} / \mathrm{min}$ per $1.73 \mathrm{~m}^{2 \prime}$ as the covariate $(\mathrm{N}=357)$

\begin{tabular}{|c|c|c|c|}
\hline & Crude & Model I & Model 2 \\
\hline \multicolumn{4}{|c|}{ eGFR $\geq 60 \mathrm{~mL} / \mathrm{min}$ per $1.73 \mathrm{~m}^{2}$} \\
\hline Urinary NAG (IU/L) & $1.15(1.03-1.73)$ & $1.08(0.93-1.58)$ & $1.04(0.89-1.47)$ \\
\hline$P$ value & 0.037 & 0.053 & 0.157 \\
\hline Urinary NAG (U/g creatinine) & $1.06(0.94-1.87)$ & $1.04(0.92-1.84)$ & $1.02(0.88-1.73)$ \\
\hline$P$ value & 0.146 & 0.205 & 0.226 \\
\hline \multicolumn{4}{|c|}{ eGFR $<60 \mathrm{~mL} / \mathrm{min}$ per $1.73 \mathrm{~m}^{2}$} \\
\hline Urinary NAG (IU/L) & $1.53(1.29-2.93)$ & $1.53(1.29-2.81)$ & $1.50(1.27-2.96)$ \\
\hline$P$ value & 0.010 & 0.011 & 0.016 \\
\hline Urinary NAG (U/g creatinine) & $1.56(1.19-2.69)$ & $1.49(1.17-2.52)$ & $1.44(1.15-2.49)$ \\
\hline$P$ value & 0.006 & 0.008 & 0.010 \\
\hline
\end{tabular}

Notes: Model I: Adjusted for duration of diabetes. Model 2: Adjusted for age, gender, smoking history, drinking history, BMl, duration of diabetes and duration of diabetes. Model 3: Adjusted for age, gender, smoking history, drinking history, BMl, systolic blood pressure, diastolic blood pressure, moderate physical activity and duration of diabetes. Abbreviations: NAG, N-acetyl- $\beta$-d-glucosaminidase; CV, cardiovascular; BMI, body mass index; eGFR, estimated glomerular filtration rate. 
Table 5 ROC Analysis of Predicting CV Events in Patients with Type 2 Diabetes Mellitus (N=357)

\begin{tabular}{|l|c|c|c|c|}
\hline Variables & AUC & Standard Error & 95\% Cl & P value \\
\hline Estimated GFR (mL/min per $\left.1.73 \mathrm{~m}^{2}\right)$ & 0.74 & 0.03 & $0.65-0.93$ & 0.012 \\
Urinary NAG (IU/L) & 0.78 & 0.04 & $0.68-0.96$ & 0.009 \\
Urinary NAG (U/g creatinine) & 0.81 & 0.05 & $0.72-0.97$ & $<0.001$ \\
\hline
\end{tabular}

Abbreviations: NAG, N-acetyl- $\beta$-d-glucosaminidase; CV, cardiovascular; eGFR, estimated glomerular filtration rate; AUC, area under the curve.

renal injury on predicting risk of $\mathrm{CV}$ events have also been investigated. ${ }^{11-13}$ As a lysosomal enzyme, NAG is highly expressed in renal tubules and its urine levels is increased in nephropathy. Increased urine level of NAG is a sensitive biomarker for renal tubular damage because the molecular mass $(130,000)$ of the protein precludes glomerular filtration. ${ }^{20,21}$ Our study demonstrated that elevated urine NAG levels were associated with a higher risk of CV events in diabetic patients. The significant association in regression models suggested that urine NAG may have an ideal utility to predict the risk of $\mathrm{CV}$ events in diabetic patients. Additionally, we also found that "abnormal renal function" has an effect modification on the association between urine NAG levels and the risk of CV events.

In previous findings, NAG values are elevated with worsening renal function and increased diabetes duration. They have concluded that urine NAG level is helpful in the diagnosis of diabetic nephropathy. ${ }^{22,23}$ Recently, the attention to markers of renal tubular damage as potential indicators for CVD is increasing rapidly. Evidence demonstrated that urinary levels of NAG are related to different CVD in various subjects. For instance, the GISSI-prevenzione trial suggested that increased urinary NAG was strongly associated with poor outcomes in population with chronic $\mathrm{HF}^{24}$ Weitgasser et al reported a significant relationship between urinary NAG and macrovascular disease in old patients with type 2 diabetes mellitus. ${ }^{14}$ Ouchi et al suggested that elevated levels of urinary NAG were associated with increased arterial stiffness in subjects without diabetes. ${ }^{25}$ In a study with a general population, urinary NAG was independently associated with high risks of ischemic stroke, myocardial infarction and all-cause mortality. Importantly, they did not found that traditional $\mathrm{CV}$ risk factors such as eGFR and albumin were associated with $\mathrm{CV}$ events when urinary NAG compared. ${ }^{26}$ Consistently, increased urinary NAG levels were significantly associated with CV events in diabetic patients in our study. Differently, our study showed that elevated urinary NAG levels were associated with higher $\mathrm{CV}$ risk in diabetic patients with abnormal renal function but not in those subjects with normal renal function. "Abnormal renal function" has an effect modification on the association between urine NAG levels and the risk of $\mathrm{CV}$ events. The most likely explanation is that renal injury leads to abnormal renal function, aggravating cardiovascular events and increasing urine levels.

This study has some notable strengths. Firstly, our findings in this prospective cohort study can further provide clinical evidence that urinary levels of NAG contributes to elevated risk of $\mathrm{CV}$ events in diabetic patients. Secondly, we reported that urinary NAG, a renal tubulopathic marker, is more closely associated with $\mathrm{CV}$ events only in diabetic patients with abnormal renal function, suggesting that diabetic nephropathy can mediate the correlation between urinary NGA levels and CV events. Thirdly, although this is a single center clinical study, we collected a relatively large number of study samples $(\mathrm{N}=$ 357), which enhances the reliability of the results. Finally, sufficient clinical confounding factors were also adjusted to further confirm the stability of our results. However, our study also has several limitations. For instance, although some confounders, including renal function, were adjusted, which may be a very important determinant of urinary NAG levels, other confounders cannot be fully excluded because many determinants of urinary NAG are currently unknown. Similar to some other biomarkers of renal injury, urinary NAG can change substantially over time. We could not perform a repeated measures analysis in this cohort due to the measurement of urinary NAG only at a single time point, which may introduce some bias into our results. The follow-up durations of variables may have affected the observed results. Furthermore, the application of a Cox regression model using a relatively large number of covariates might cause overfitting of the model.

\section{Conclusions}

We provided the evidence that elevated urinary NAG levels were related to risk of $\mathrm{CV}$ outcomes in diabetic patients. The "abnormal renal function" has an effect 
modification on the association between urine NAG levels and the risk of $\mathrm{CV}$ events. These results might further suggested that urinary NAG is a value urinary biomarker for early detecting $\mathrm{CV}$ events.

\section{Funding}

There is no funding to report.

\section{Disclosure}

The authors report no conflicts of interest in this work.

\section{References}

1. Hosh-Swaby OR, Goodman SG, Leiter LA, et al. Glucose-lowering drugs or strategies, atherosclerotic cardiovascular events, and heart failure in people with or at risk of type 2 diabetes: an updated systematic review and meta-analysis of randomised cardiovascular outcome trials. Lancet Diabetes Endocrinol. 2020;8(5):418-435. doi:10.1016/S2213-8587(20)30038-3

2. Pollock C, Wheeler DC, Zhang H, Zinman B, Mahaffey KW. Evaluating the effects of canagliflozin on cardiovascular and renal events in patients with type 2 diabetes mellitus and chronic kidney disease according to baseline $\mathrm{HbAlc}$, including those with $\mathrm{HbA1c}$ $<7 \%$ : results from the CREDENCE trial. Circulation. 2020;141 (5):407-410.

3. Wanner C, Lachin JM, Inzucchi SE, et al; EMPA-REG OUTCOME Investigators. Empagliflozin and clinical outcomes in patients with type 2 diabetes mellitus, established cardiovascular disease, and chronic kidney disease. Circulation. 2018;137(2):119-129. doi:10.1161/CIRCULATIONAHA.117.028268

4. Eckel RH, Kahn R, Robertson RM, Rizza RA. Preventing cardiovascular disease and diabetes: a call to action from the American Diabetes Association and the American Heart Association. Circulation. 2006;113(25):2943-2946. doi:10.1161/ CIRCULATIONAHA.106.176583

5. Mulè G, Cottone S, Cusimano $\mathrm{P}$, et al. The association of microalbuminuria with aortic stiffness is independent of $\mathrm{C}$-reactive protein in essential hypertension. Am J Hypertens. 2009;22(10):1041-1047. doi:10.1038/ajh.2009.132

6. Ishimura E, Taniwaki H, Tsuchida T, et al. Urinary albumin excretion associated with arterial wall stiffness rather than thickness in type 2 diabetic patients. $J$ Nephrol. 2007;20(2):204-211.

7. Savage S, Estacio RO, Jeffers B, Schrier RW. Urinary albumin excretion as a predictor of diabetic retinopathy, neuropathy, and cardiovascular disease in NIDDM. Diabetes Care. 1996;19 (11):1243-1248. doi:10.2337/diacare.19.11.1243

8. Willeit P, Tschiderer L, Allara E, et al; PROG-IMT and the ProofATHERO Study Groups. Carotid intima-media thickness progression as surrogate marker for cardiovascular risk: meta-analysis of 119 clinical trials involving 100667 patients. Circulation. 2020;142 (7):621-642. doi:10.1161/CIRCULATIONAHA.120.046361

9. Mulè $\mathrm{G}$, Cottone S, Vadalà $\mathrm{A}$, et al. Relationship between albumin excretion rate and aortic stiffness in untreated essential hypertensive patients. J Intern Med. 2004;256(1):22-29. doi:10.1111/j.13652796.2004.01338.x

10. Huang Y, Chen Y, Xu M, et al. Low-grade albuminuria is associated with carotid intima-media thickness in Chinese type 2 diabetic patients. J Clin Endocrinol Metab. 2010;95(11):5122-5128. doi:10.1210/jc.2010-0544
11. Kim SR, Lee YH, Lee SG, et al. Urinary N-acetyl- $\beta$ D-glucosaminidase, an early marker of diabetic kidney disease, might reflect glucose excursion in patients with type 2 diabetes. Medicine. 2016;95(27):e4114. doi:10.1097/MD.0000000000004114

12. Lee M, Hong N, Lee YH, Kang ES, Cha BS, Lee BW. Elevated $\mathrm{N}$-acetyl- $\beta$-d-glucosaminidase, a urinary tubular damage marker, is a significant predictor of carotid artery atherosclerosis in type 1 diabetes, independent of albuminuria: a cross-sectional study. $J$ Diabetes Complications. 2018;32(8):777-783. doi:10.1016/j. jdiacomp.2018.05.019

13. Auta FL, Boertien WE, Bakker SJ, et al. Glomerular and tubular damage markers are elevated in patients with diabetes. Diabetes Care. 2011;34(4):975-981. doi:10.2337/dc10-1545

14. Weitgasser R, Schnoell F, Gappmayer B, Kartnig I. Prospective evaluation of urinary $\mathrm{N}$-acetyl-beta-D-glucosaminidase with respect to macrovascular disease in elderly type 2 diabetic patients. Diabetes Care. 1999;22(11):1882-1886. doi:10.2337/diacare.22.11.1882

15. Kopf S, Oikonomou D, Zdunek D, et al. Urinary n-acetyl-beta-d-glucosaminidase excretion: an indicator of neuropathy in type 2 diabetes. Exp Clin Endocrinol Diabetes. 2013;121(10):601-606. doi:10.1055/ s-0033-1355384

16. Shimojo N, Kitahashi S, Naka K, et al. Comparison of N-acetyl-beta -D-glucosaminidase and alanine aminopeptidase activities for evaluation of microangiopathy in diabetes mellitus. Metabolism. 1987;36 (3):277-280. doi:10.1016/0026-0495(87)90188-0

17. Gloyn AL, Drucker DJ. Precision medicine in the management of type 2 diabetes. Lancet Diabetes Endocrinol. 2018;6(11):891-900. doi:10.1016/S2213-8587(18)30052-4

18. Bikbov B, Purcell CA, Levey AS; GBD Chronic Kidney Disease Collaboration. Global, regional, and national burden of chronic kidney disease, 1990-2017: a systematic analysis for the Global Burden of Disease Study 2017. Lancet. 2020;395(10225):709-733. doi:10.1016/S0140-6736(20)30045-3

19. Laffel LM, Kanapka LG, Beck RW, et al; CGM Intervention in Teens and Young Adults with T1D (CITY) Study Group; CDE10. Effect of continuous glucose monitoring on glycemic control in adolescents and young adults with type 1 diabetes: a randomized clinical trial. JAMA. 2020;323(23):2388-2396. doi:10.1001/jama.2020.6940

20. Price RG. The role of NAG (N-acetyl-beta-D-glucosaminidase) in the diagnosis of kidney disease including the monitoring of nephrotoxicity. Clin Nephrol. 1992;38(Suppl 1):S14-s19.

21. García-Nieto V, García-Rodríguez VE, Luis-Yanes MI, Monge M, Arango-Sancho P, Garin EH. Renal tubular markers as screening tools for severe vesicoureteral reflux. Eur J Pediatr. 2019;178 (4):525-531. doi:10.1007/s00431-019-03324-9

22. Kordonouri O, Hartmann R, Müller C, Danne T, Weber B. Predictive value of tubular markers for the development of microalbuminuria in adolescents with diabetes. Horm Res. 1998;50(Suppl 1):23-27.

23. Kato H, Takashima T, Kishikawa H, Emura S, Ohmori K. The significance of urinary N-acetyl-beta-D-glucosaminidase for predicting early stage diabetic nephropathy. Int J Clin Pract. 1997;51 (8):489-490.

24. Damman K, Masson S, Hillege HL, et al. Clinical outcome of renal tubular damage in chronic heart failure. Eur Heart J. 2011;32 (21):2705-2712. doi:10.1093/eurheartj/ehr190

25. Ouchi M, Oba K, Saigusa T, et al. Association between pulse wave velocity and a marker of renal tubular damage (N-acetyl- $\beta$ D-glucosaminidase) in patients without diabetes. $J$ Clin Hypertens. 2015;17(4):290-297. doi:10.1111/jch.12492

26. Solbu MD, Toft I, Lochen ML, Mathiesen EB, Eriksen BO, Melsom T. N-Acetyl- $\beta$-d-glucosaminidase does not enhance prediction of cardiovascular or all-cause mortality by albuminuria in a lowrisk population. $J$ Am Soc Nephrol. 2016;27(2):533-542. doi:10.1681/ASN.2014100960 


\section{Publish your work in this journal}

The International Journal of General Medicine is an international, peer-reviewed open-access journal that focuses on general and internal medicine, pathogenesis, epidemiology, diagnosis, monitoring and treatment protocols. The journal is characterized by the rapid reporting of reviews, original research and clinical studies across all disease areas. The manuscript management system is completely online and includes a very quick and fair peer-review system, which is all easy to use. Visit http://www.dovepress.com/ testimonials.php to read real quotes from published authors.

Submit your manuscript here: https://www.dovepress.com/international-journal-of-general-medicine-journal 